\title{
Biologika in der Therapie kindlicher chronisch-entzündlicher Darmerkrankungen
}

Kinder und Jugendliche mit chronisch-entzündlichen Darmerkrankungen (CED) leiden unter einer erheblichen Langzeit-Morbidität. Longitudinalstudien zeigen, dass über 50\% der Kinder mit Morbus Crohn nach 10 Jahren operiert werden müssen. Hauptindikationen für eine Operation sind Stenosen und Fisteln, während die entzündliche Komponente im Verlauf abnimmt. Darüber hinaus ist bekannt, dass die Aktivität der CED bei Kindern häufig ausgeprägter ist als bei Erwachsenen. Daher benötigen Kinder und Jugendliche eine besonders effiziente CED-Therapie. Medikamente wie Steroide, die häufig bei Erwachsenen mit CED eingesetzt werden, führen zu schweren Nebenwirkungen wie Wachstumsstörungen und Osteoporose, fördern die Fistelbildung und werden deshalb im Rahmen der Therapie des kindlichen Morbus Crohn vermieden. Biologika, die in der CED-Therapie eingesetzt werden, sind monoklonale Antikörper gegen
Entzündungsmediatoren (TNF $\alpha$, Interleukin-12/23) oder Adhäsionsmoleküle ( $\alpha 4 \beta 7$ Integrin). Monoklonale Antikörper gegen Adhäsionsmoleküle und Interleukin-12/23 sind noch nicht für die Therapie der kindlichen CED zugelassen und werden daher nur im Rahmen von Studien oder nach Einzelfallgenehmigung verabreicht. Biologika werden häufig mit immunmodulatorischen Medikamenten wie Methotrexat oder Thiopurinen kombiniert. Bei ausgeprägter Krankheitsaktivität werden Biologika auch primär eingesetzt („topdown“-Therapiestrategie). Die meiste Erfahrung in der Biologika-Therapie der kindlichen CED besteht mit TNF $\alpha$-Blockern. Die Remissionsrate liegt nach einem Jahr bei $50-60 \%$ mit einem etwas schlechteren Ansprechen der Colitis ulcerosa im Vergleich zu Morbus Crohn. Therapeutisches Drug-Monitoring mit Messung der Wirkspiegel und AntiDrug-Antikörper im Serum ist essenziell für die optimale Steuerung der Therapie.
Interessenkonflikte

Der Autor hat Vortragshonorare von AbbVie und Nutricia erhalten.

Autor

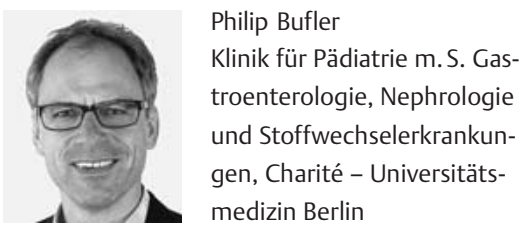

Korrespondenzadresse

Prof. Dr. med. Philip Bufler

Klinik für Pädiatrie m. S. Gastroenterologie, Nephrologie und Stoffwechselerkrankungen Charité - Universitätsmedizin Berlin Campus Virchow-Klinikum - CC17 Augustenburger Platz 1 13353 Berlin

philip.bufler@charite.de 